\title{
Contributions of temporal and place cues in pitch perception in absolute pitch possessors
}

\author{
WAKA FUJISAKI and MAKIO KASHINO \\ NTT Communication Science Laboratories, Atsugi, Japan
}

\begin{abstract}
Pitch perception is determined by both place and temporal cues. To explore whether the manner in which these cues are used differs depending on absolute pitch capability, pitch identification experiments with and without pitch references were conducted for subjects with different absolute pitch capabilities and musical experience. Three types of stimuli were used to manipulate place and temporal cues separately: narrowband noises, which provide strong place cues but less salient temporal cues; iterated rippled noises, which provide strong temporal cues but less salient place cues; and sinusoidal tones, which provide both. The results indicated that absolute pitch possessors utilize temporal cues more effectively when they identify musical chroma. With regard to the judgment of height, it was indicated that place cues play an important role for both absolute and nonabsolute pitch possessors.
\end{abstract}

A person with absolute pitch (AP) can identify or produce the pitch of a sound without hearing a reference sound (Bachem, 1937; Takeuchi \& Hulse, 1993). It is generally thought that AP possessors are superior in all of their auditory and musical abilities. However, in the field of auditory and music psychology, researchers have focused mainly on the etiology of AP (Brady, 1970; Cuddy, 1968; Profita \& Bidder, 1988), the merits and demerits of AP in music cognition (Bachem, 1955; Benguerel \& Westdal, 1991; Miyazaki, 1993, 1995; Miyazaki \& Rakowski, 2002; Siegel, 1972), or the neural characteristics of AP (Klein, Coles, \& Donchin, 1984; Wayman, Frisina, Walton, Hantz, \& Crummer, 1992; Zatorre, 2003); they have not closely studied the basic hearing mechanisms of AP possessors for general sounds outside the context of music.

Studying the basic hearing abilities of AP possessors may provide insights not only into the mechanisms of AP itself, but also into problems, such as diversity and the plasticity of auditory functions. These possibilities led us to conduct a series of experiments to examine whether AP capability has any influence on basic hearing abilities (Fujisaki \& Kashino, 2002). The overall results showed that there were no significant differences in frequency, temporal, and spatial resolutions among groups with different AP capabilities.

The next question is, then, the following: Where do the differences exist between AP and non-AP (NAP) possessors? Since AP is the ability to identify musical pitch, is it possible for differences from non-AP to exist in mechanisms directly related to musical pitch perception?

The main part of this article is based on W.F.'s doctoral thesis at Ochanomizu University, Tokyo. Portions of this article were published in the Journal of the Acoustical Society of Japan, 57(12), in Japanese. Correspondence should be addressed to W. Fujisaki, NTT Communication Science Laboratories, NTT Corporation, 3-1 Morinosato-Wakamiya, Atsugi, 243-0198 Japan (e-mail: fujisaki@avg.brl.ntt.co.jp).
The major cues for pitch perception are place cues and temporal cues (Moore, 1997). Sound waves reach the ear and cause the basilar membrane located in the cochlea of the inner ear to vibrate. The peak of the vibration is near the end of the basilar membrane when frequencies are low and near the entrance (the oval window) when frequencies are high. Inner hair cells on the basilar membrane transform the vibration into auditory nerve fiber impulses. More impulses are produced when the vibrations are large. Therefore, the amplitude spectrum of an input signal reaching the inner ear can be coded by the excitation pattern of auditory nerve fibers along the basilar membrane. This information is called a place cue because it is based on the location of the vibration on the basilar membrane. On the other hand, each auditory nerve fiber discharges impulses synchronized with a certain phase of the basilar membrane vibrations when frequencies are below 4-5 kHz. Therefore, the periodicity of impulses that each auditory nerve fiber discharges contains information about the frequency component of the input signal. This information is called a temporal cue. In the case of a complex sound, such as the sound of a musical instrument or a human voice, both cues work together to provide information that is used to determine pitch. However, it is still unclear whether the manner in which these cues are used differs depending on AP capability and musical experience.

In this study, we conducted two experiments to answer these questions. In the first experiment, subjects performed relative pitch identification tasks in which a reference sound was presented before each target stimulus. In these tasks, place and temporal cues were separately manipulated. Then the results across groups with different degrees of absolute pitch were compared. We conducted relative pitch identification tasks, rather than AP identification tasks, because NAP possessors have difficulty performing in AP identification tasks. In the second experi- 
ment, to examine how AP possessors use place and temporal cues when they identify musical pitch, subjects performed AP identification tasks in which these cues were separately manipulated. No reference sound was presented before each target stimulus. AP and partial absolute pitch (PAP) possessors participated in the second experiment.

\section{EXPERIMENT 1}

\section{Method}

Subjects. The subjects were 12 female musicians and 6 female nonmusicians with normal hearing. The musicians had at least 8 years of formal musical training before the age of 20 years. The nonmusicians had less than 2 years of musical experience. The subjects were from 18 to 32 years of age and were paid for their participation.

Before the main experiment, an AP test was conducted. Pure tones in three octaves were presented in random order, with the constraint that tones within the same octaves or tones having the same chroma were never presented successively. The duration of each tone was $200 \mathrm{msec}$. The reason for not presenting two consecutive stimuli within the same octave was to prevent the subjects from using relative pitch when they made judgments and, thus, make the test more challenging. Likewise, a short duration of the stimuli was used to reduce the chance of the subjects' using relative pitch. Prior to the AP test, it was confirmed that all the subjects knew the correspondence between keys of the musical keyboard and musical pitch names. The subjects identified and chose the musical pitch by clicking keys on a computer screen. The keys were displayed in the same order as that on a musical keyboard. When the subjects could not identify the pitch name of the target sound, they were instructed to choose the key that they thought was closest to the target sound. The next stimulus was presented $3 \mathrm{sec}$ after a response. One session contained 36 trials, and at least three sessions were conducted for each subject. On the basis of the results of the AP test, the subjects were divided into three groups: AP musicians, NAP musicians, and NAP nonmusicians (NMs). AP subjects were musicians who scored more than $97 \%$ on the absolute pitch test, NAP subjects were musicians who scored under $25 \%$, and NM subjects were nonmusicians who scored under $25 \%$. Six of the musicians were classified as AP subjects, and the other six as NAP subjects. The six nonmusicians were classified as NM subjects.
Stimuli and Equipment. Figure 1 shows some examples of sound spectrograms of stimuli used in this experiment. Three types of stimuli were used for pitch identification tasks: narrowband noises (NBNs), in which pitch perception is produced mainly by place cues and temporal cues are rather less salient (Figure 1A); repeated broadband noises with a designated delay and gain and limited bandwidth from 1000 to $3500 \mathrm{~Hz}$ (iterated rippled noises [IRNs]), in which pitch perception is produced mainly by temporal cues and place cues are rather less salient (Figure 1B); and pure tones (PTs), in which pitch perception is produced by both place and temporal cues (Figure 1C). The bandwidth of the NBNs was 50 cents, and the slope was $200 \mathrm{~dB}$ /octave. IRNs, unlike NBNs, contain regular periodic information. IRNs with $1 / d-\mathrm{kHz}$ pitch can be designed by repeating the process of adding noise with $d$-msec delay $(100-\mathrm{Hz}$ pitch is perceived with $10-\mathrm{msec}$ delay), by adding the original noise each time (add-original network) or adding the latest repeated noise (add-same network; Yost, 1996). In this experiment, we used the latter approach. This noise-adding process was repeated 16 times, and the gain of the repeated noise was about 0.52 of the latest repeated noise.

Although there are some spectral ripples in IRN stimuli, they are hard for the auditory system to resolve in high-frequency regions. The bandwidth of the IRNs was limited to $1000-3500 \mathrm{~Hz}$ so that resolvable low-frequency components were filtered out from the stimuli. IRN spectral ripples still remained in the $1000-3500-\mathrm{Hz}$ regions; however, they were contiguous and relatively weak. Therefore, temporal cues were stronger and reliable, although spectral information was not fully eliminated.

The signals were $250 \mathrm{msec}$ in duration and were shaped with 10msec cosine ramps. The overall level of the stimuli, presented diotically via headphones (Sennheiser HDA 200), was about 60 dB SPL. The stimuli were generated digitally and sent to a D/A converter (STAX DAC-TALENT) through a sound card (Digital Audio Labs: digital I/O card) on a computer (Gateway 2000 GP6-400).

Procedure. The task was to identify the note name of a target sound by pressing the corresponding keys on the computer screen. The task was almost the same as in the AP test, but a reference sound (middle C, $261.6 \mathrm{~Hz}$ ) was presented before each target stimulus. The range of the target stimulus was $\mathrm{C} 2(65.4 \mathrm{~Hz})$ to B6 $(1975.5 \mathrm{~Hz})$.

Target stimuli in five octaves were presented in random order, with the constraint that tones within the same octaves or tones having the same chroma were never presented successively. No feedback

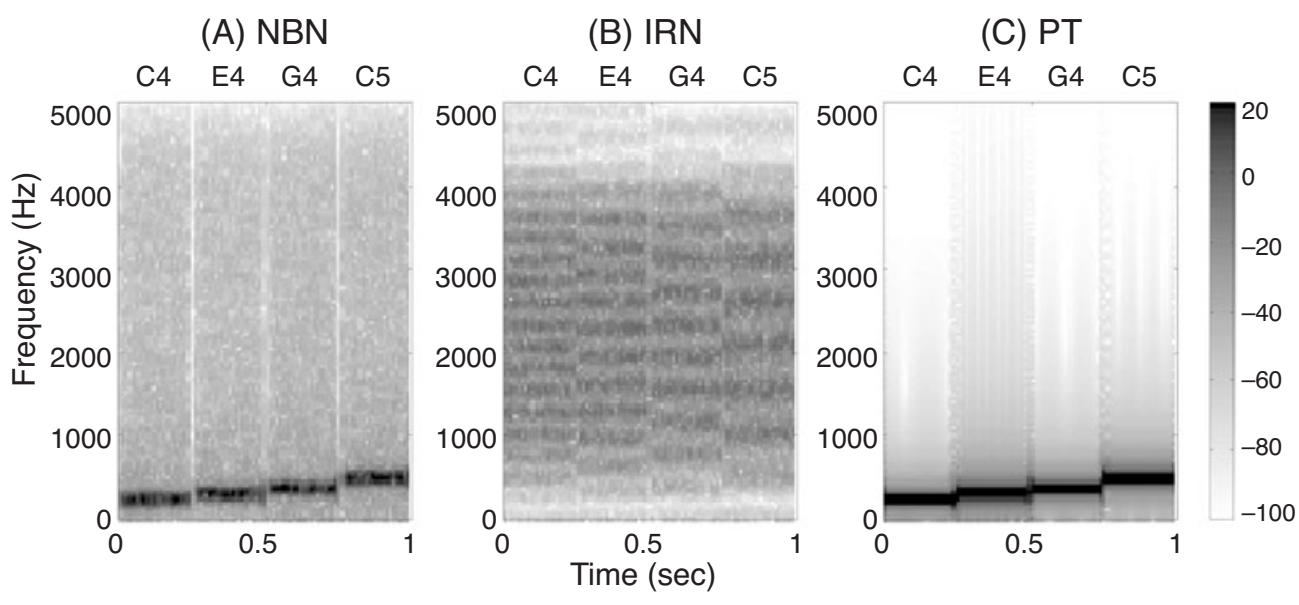

Figure 1. Sound spectrograms of the stimuli used in Experiments 1 and 2. Brightness represents the amplitude of the spectral components. Darker parts represent higher amplitude (in relative decibels). The horizontal axis represents time (in seconds), and the vertical axis represents frequency (in hertz). C4, E4, G4, and $C 5$ notes are shown every $0.25 \mathrm{sec}$. Spectrograms indicate (A) narrowband noises (NBNs), (B) iterated rippled noises (IRNs), and (C) pure tones (PTs). 
was provided. The order of stimuli conditions (NBN, IRN, PT) was randomized for each subject. One trial sequence contained 60 trials in which notes from five octaves including all 12 chromatic tones were presented. There were three trial sequences in each condition for each subject, and the last two trial sequences were used for the analysis.

\section{Results and Discussion}

The numbers of correct responses were defined in three ways. Chroma correct responses were defined as responses in which the note names of the presented target sounds and the subjects' responses matched, ignoring absolute height (chance level was about 8.3\%). Height correct responses were defined as the responses in which the note names of the target sounds and the responses were within \pm 5 semitones (chance level was about $18.3 \%$ ). Chroma and height correct responses were the responses in which the note names of the target sounds and the responses matched exactly, including absolute height (chance level was about 1.6\%). Chroma correct and height correct scores were analyzed separately. We did not specifically analyze "chroma and height" correct scores, since we had already conducted individual analyses of these scores, but the calculation results are shown in Figure 4.

Comparisons of chroma identification among groups. Chroma correct scores in Experiment 1 are shown in Figure 2. They were analyzed with a two-way analysis of variance (ANOVA) to examine the effects of groups (AP, NAP, and NM) and stimulus conditions (NBN, IRN, and PT). The interaction between groups and stimulus conditions was significant $[F(4,30)=14.77, p<.01]$.

Since the interaction between groups and stimulus conditions was significant, the simple main effects for each factor were analyzed. First, the simple main effects for groups were analyzed. In all groups, a significant effect of stimulus conditions was found [AP, $F(2,10)=23.78, p<$ $.01 ; \mathrm{NAP}, F(2,10)=4.67, p<.05 ; \mathrm{NM}, F(2,10)=23.24$, $p<.01]$. From Tukey's HSD test on stimuli, in the AP group, there were significant differences between PTs and
NBNs $(p<.01)$ and between IRNs and NBNs $(p<.01)$. There was no significant difference between PTs and IRNs. In the NAP group, there was a significant difference between PTs and NBNs $(p<.05)$. In the NM group, there were significant differences between PTs and IRNs $(p<.01)$ and between PTs and NBNs $(p<.01)$.

Next, simple main effects for stimuli were analyzed. In all the stimulus conditions, there were significant effects of groups [PTs, $F(2,15)=60.23, p<.01$; IRNs, $F(2,15)=113.57, p<.01 ; \mathrm{NBNs}, F(2,15)=34.29, p<$ $.01]$. From Tukey's HSD test on groups, there were significant differences between AP and NAP (PTs, $p<.01$; IRNs, $p<.01$; NBNs, $p<.05)$, between AP and NM $(p<.01$ in all the conditions), and between NAP and NM ( $p<.01$ in all the conditions).

NBNs provide strong place cues but less salient temporal cues, and IRNs provide strong temporal cues and less salient place cues. Therefore, the results in which chroma scores declined for the NBN condition and did not decline for the IRN condition in the AP group suggest that temporal cues are very important for chroma identification for AP possessors.

On the other hand, the chroma score declined in both the NBN and the IRN conditions in the NM group. This suggests that it was difficult for NM subjects to identify chroma when only temporal cues were presented.

The results for NAP musicians were in between. The chroma scores significantly declined for the NBN condition, as compared with the PT condition. However, there were no significant differences between NBNs and IRNs nor between IRNs and PTs. This suggests that NAP musicians put more weight on temporal cues than do NMs and less weight on temporal cues than do AP musicians when they identify chroma.

Comparisons of height identification among groups. Figure 3 shows the results of height identification. Height correct scores were analyzed with a two-way ANOVA. The main effects of both groups (AP, NAP, and

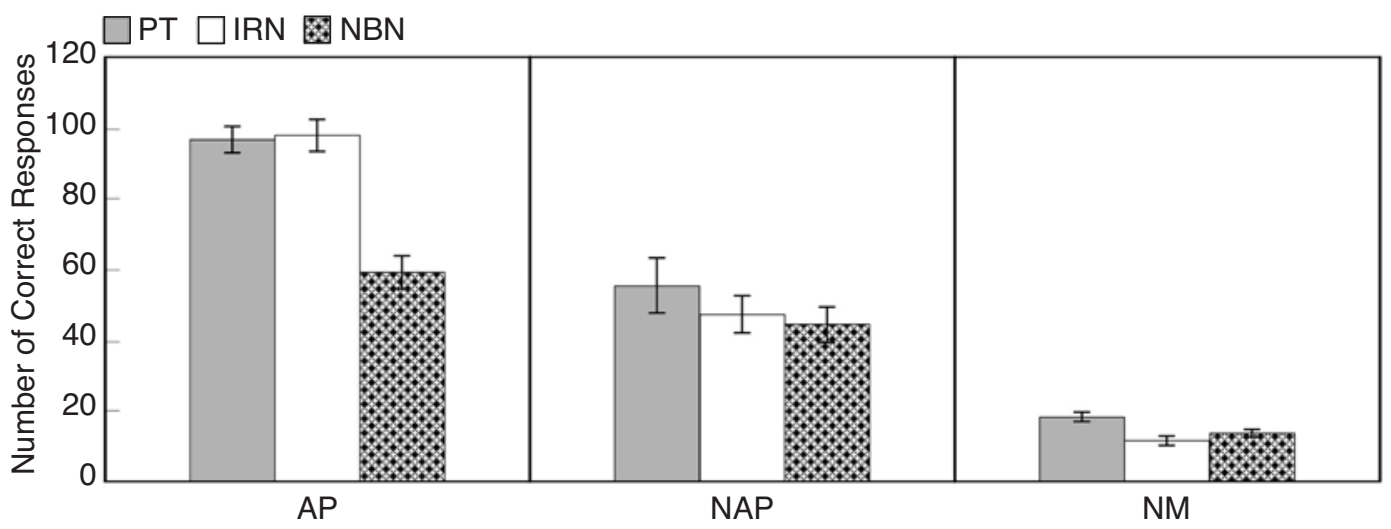

Figure 2. Chroma identification scores in Experiment 1. The numbers of correct responses in chroma identification with pure tone (PT), iterated rippled noise (IRN), and narrowband noise (NBN) stimuli for absolute pitch (AP) musicians, nonabsolute pitch (NAP) musicians, and nonabsolute pitch nonmusicians (NMs) are shown. Error bars represent $\pm 1 S E M$. A reference sound (middle $C, 261.6 \mathrm{~Hz}$ ) as in Figures 3 and 4 was presented before each target stimulus. 


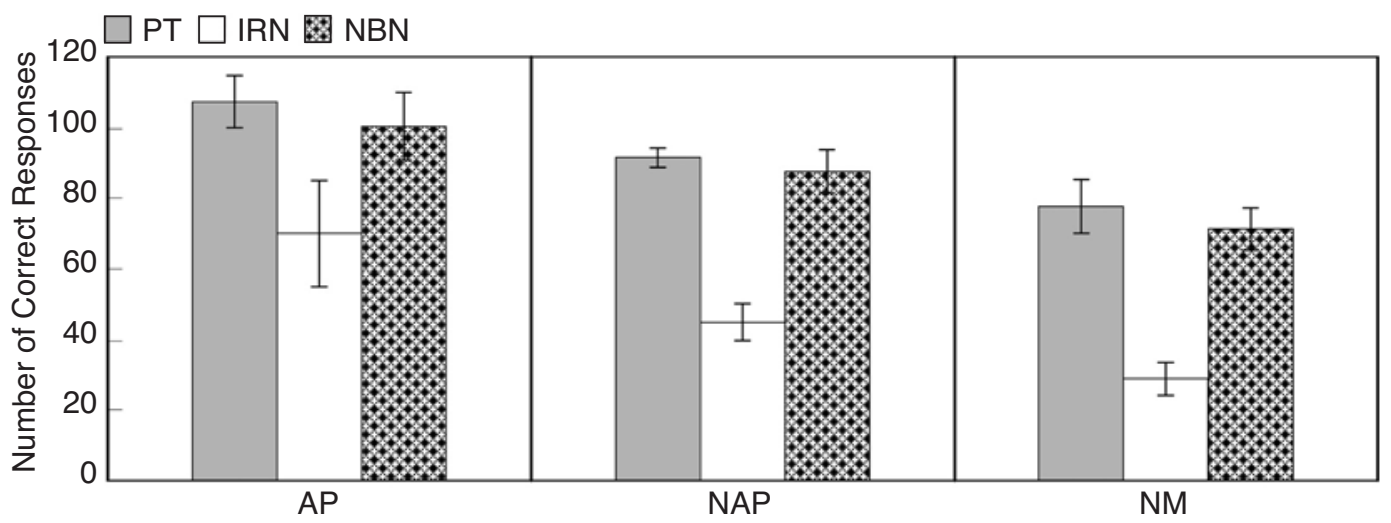

Figure 3. Height identification scores in Experiment 1. The numbers of correct responses in height identification with pure tone (PT), iterated rippled noise (IRN), and narrowband noise (NBN) stimuli for absolute pitch musicians (AP), nonabsolute pitch musicians (NAP), and nonabsolute pitch nonmusicians (NMs) are shown. Error bars represent $\pm 1 S E M$. A reference sound (middle $C, 261.6 \mathrm{~Hz}$ ) was presented before each target stimulus.

$\mathrm{NM})$ and stimulus conditions (NBN, IRN, and PT) were significant [groups, $F(2,15)=6.50, p<.01$; stimulus conditions, $F(2,30)=59.49, p<.01]$. The interaction between groups and conditions was not significant. From Tukey's HSD test on groups, there was a significant difference between the AP group and NMs $(p<.01)$. No significant differences were found between the AP and the NAP groups or between the NAP and the NM groups. From Tukey's HSD test on stimulus conditions, there were significant differences between NBNs and IRNs $(p<.01)$ and between IRNs and PTs $(p<.01)$. No differences were found between PTs and NBNs.

IRNs provide strong temporal cues but less salient place cues, and NBNs provide strong place cues but less salient temporal cues. Therefore, the fact that the height score declined for the IRN condition, but not for the NBN condition, suggests that place cues play an important role in height identification.
Planned comparisons for the effect of groups. To analyze the effect of AP and musical experience separately, planned comparisons were conducted. First, the effect of AP was analyzed. The AP group was compared with the NAP and the NM groups in each stimulus condition. For chroma identification scores, there were significant differences between groups in all the stimulus conditions [PTs, $F(1,15)=93.37, p<.01$; IRNs, $F(1,15)=$ $188.60, p<.01$; NBNs, $F(1,15)=38.36, p<.01]$. For height identification scores, the effects of groups were also found in all stimulus conditions [PTs, $F(1,15)=$ $8.59, p<.05$; IRNs, $F(1,15)=7.98, p<.05$; NBNs, $F(1,15)=5.34, p<.05]$.

Next, to examine the effect of musical experiences, we compared the AP and the NAP groups with the NM group. In chroma identification scores, there were significant differences between groups for all the stimulus conditions $[\mathrm{PTs}, F(1,15)=87.22, p<.01$; IRNs, $F(1,15)=$

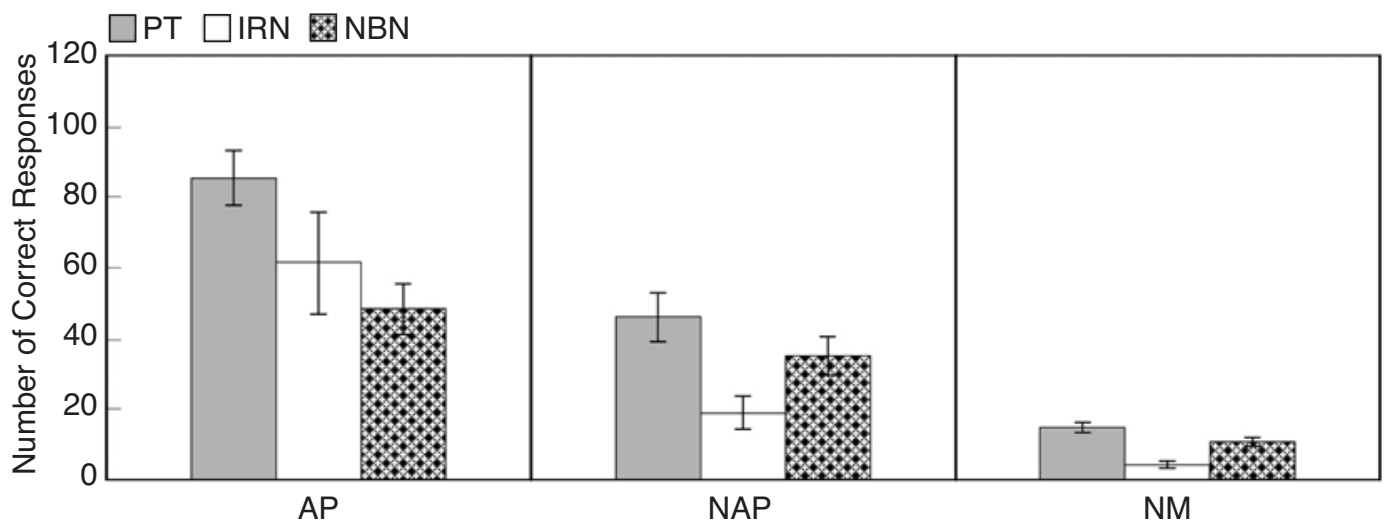

Figure 4. Chroma and height identification scores in Experiment 1. The numbers of correct responses in both chroma and height identification with pure tone (PT), iterated rippled noise (IRN), and narrowband noise (NBN) stimuli for absolute pitch (AP) musicians, nonabsolute pitch (NAP) musicians, and nonabsolute pitch nonmusicians (NMs) are shown. Error bars represent \pm 1 SEM. A reference sound (middle $C, 261.6 \mathrm{~Hz}$ ) was presented before each target stimulus. 
$149.88, p<.01$; NBNs, $F(1,15)=61.74, p<.01]$. For height identification scores, there were also significant differences between groups in all the stimulus conditions $[\mathrm{PTs}, F(1,15)=7.85, p<.05$; IRNs, $F(1,15)=5.95, p<$ .05 ; NBNs, $F(1,15)=6.27, p<.05]$.

The results obtained with the planned comparison for the effects of groups in the chroma identification showed that there were significant effects of both AP capabilities and musical experience in all stimulus conditions at the $1 \%$ level. If we take the results obtained with ANOVA and the planned comparison together, the results could be interpreted to mean that the AP possessors and the musical experts could utilize temporal cues more effectively than could the nonmusical experts when they identified musical chroma. With regard to the identification of height, although the effects were significant at the $5 \%$ level with the planned comparison, the results obtained from ANOVA did not reach significance either between AP and NAP or between NAP and NM. If we take the results obtained from ANOVA and the planned comparison together, it could be interpreted to mean that there may be some differences in the usage of place cues in height identification depending on AP capabilities and musical experience; however, the effects of groups in the height identification were not as obvious as those in the chroma identification.

\section{EXPERIMENT 2}

\section{Method}

Subjects. The subjects were 15 female musicians with normal hearing. Like the musicians in Experiment 1, they had at least 8 years of formal musical training before the age of 20 years.

On the basis of the results of the AP test, the subjects were divided into two groups: AP musicians and PAP musicians. The AP subjects were musicians who scored more than $97 \%$ on the AP test, and the PAP subjects were those who scored between $65 \%$ and $85 \%$. Eight were classified as AP subjects, and the other 7 as PAP subjects. Three of the 8 AP subjects also had participated in Experiment 1.

Stimuli and Equipment. The stimuli for pitch identification tasks and the equipment were the same as those in Experiment 1.
Procedure. The task was almost same as that in Experiment 1, but no reference sound was presented before each target stimulus. As in Experiment 1, the range of target stimulus was $\mathrm{C} 2(65.4 \mathrm{~Hz})$ to B6 $(1975.5 \mathrm{~Hz})$, and the stimuli for AP identification tasks were NBNs, IRNs, and PTs.

\section{Results and Discussion}

Figures 5-7 show the results of this experiment. As in Experiment 1, we calculated the percentage of correct responses in three ways: chroma correct, height correct, and chroma and height correct. As in Experiment 1, chroma correct and height correct scores were analyzed separately. We did not specifically analyze "chroma and height" correct scores (since we had already conducted individual analyses of these scores), but the calculation results are shown in Figure 7.

Comparisons of chroma identification between groups. Figure 5 shows the chroma correct scores in Experiment 2. Chroma correct scores were analyzed with a two-way ANOVA. The main effect for both groups (AP and PAP) and stimulus conditions (NBN, IRN, and PT) were significant [groups, $F(1,13)=24.54, p<.01$; stimulus conditions, $F(2,26)=17.56, p<.01]$. The interaction between groups and conditions was not significant. The significant effect of groups indicated that AP possessors scored higher than PAP possessors in chroma identification. From Tukey's HSD test on stimulus conditions, there were significant differences between NBNs and IRNs $(p<.01)$ and between NBNs and PTs $(p<$ .01 ). No differences were found between PTs and IRNs.

The finding that chroma score declined in the NBN condition, but not in the IRN condition, suggests that temporal cues play an important role in chroma identification for AP and PAP possessors.

Comparisons of height identification between groups. Figure 6 shows the height correct scores. Height correct scores were analyzed with a two-way ANOVA. The main effect of stimulus conditions (NBN, IRN, and PT) was significant $[F(2,26)=10.81, p<.01]$. How-

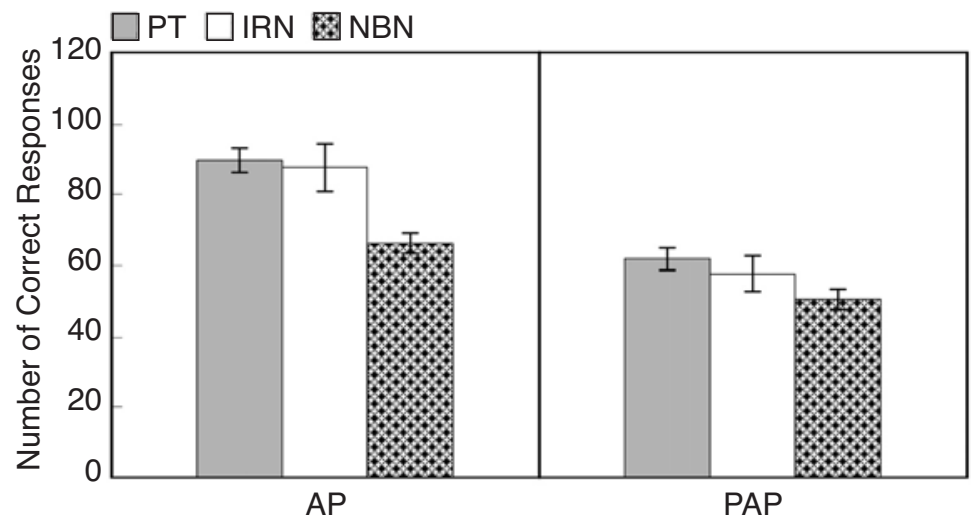

Figure 5. Chroma identification scores in Experiment 2. The numbers of correct responses in chroma identification with pure tone (PT), iterated rippled noise (IRN), and narrowband noise (NBN) stimuli for absolute pitch (AP) musicians and partial absolute pitch (PAP) musicians are shown. Error bars represent $\pm 1 S E M$. No reference sound was presented before each target stimulus. 


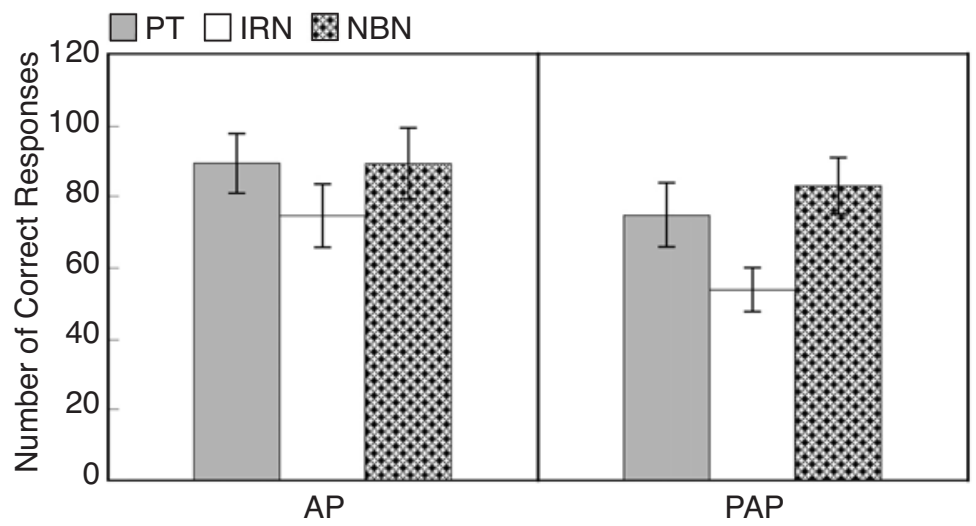

Figure 6. Height identification scores in Experiment 2. The numbers of correct responses in height identification with pure tone $(\mathrm{PT})$, iterated rippled noise (IRN), and narrowband noise (NBN) stimuli for absolute pitch (AP) musicians and partial absolute pitch (PAP) musicians are shown. Error bars represent $\pm 1 S E M$. No reference sound was presented before any target stimulus.

ever, neither the main effect of groups nor the interaction between groups and stimulus conditions was significant. The lack of any significant difference between the groups suggests that, unlike the chroma identification ability, the height identification abilities of AP possessors and PAP possessors were not significantly different.

From Tukey's HSD test on stimuli, height scores for the IRN condition were significantly lower than the scores for the $\mathrm{PT}(p<.01)$ and the NBN $(p<.01)$ conditions. The finding that the height score declined in the IRN condition, but not in the NBN condition, suggests that place cues play an important role for height identification.

Individual differences within groups. Figure 8 shows the chroma correct and the chroma and height cor- rect scores for each subject for both AP and PAP groups. The scores from each group are shown as one circle, and each axis represents the percentage of correct responses for each subject. The white square indicates chroma scores, and the black square chroma and height scores. The differences between chroma correct and chroma and height correct scores represent octave errors.

The individual response patterns within the AP and the PAP groups were not uniform. Some subjects made many octave errors, whereas others made few or none at all. This suggests that although all AP possessors can utilize temporal cues effectively when they identify chroma, the weights they put on place cues vary. There were several types throughout the AP and the PAP groups, sug-

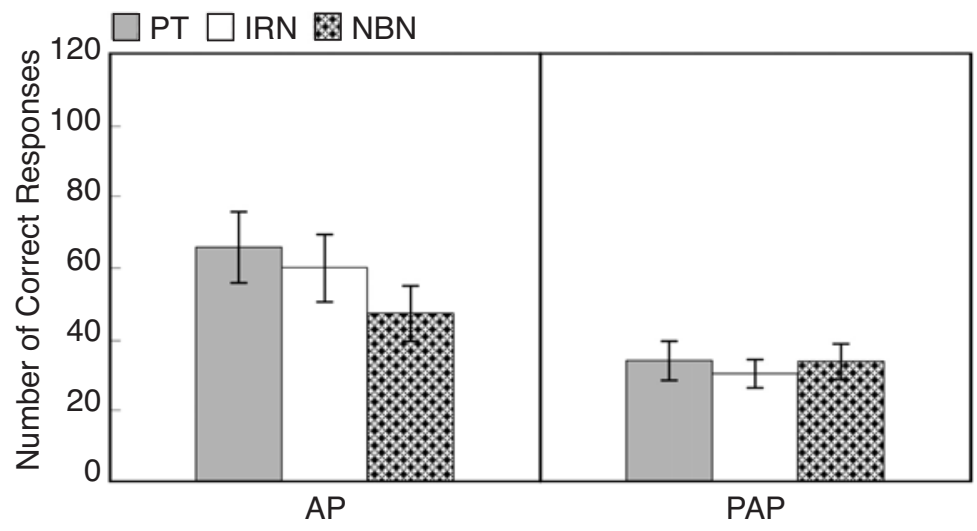

Figure 7. Chroma and height identification scores in Experiment 2. The numbers of correct responses in chroma and height identification with pure tone (PT), iterated rippled noise (IRN), and narrowband noise (NBN) stimuli for absolute pitch (AP) musicians and partial absolute pitch (PAP) musicians are shown. Error bars represent $\pm 1 S E M$. No reference sound was presented before each target stimulus. 

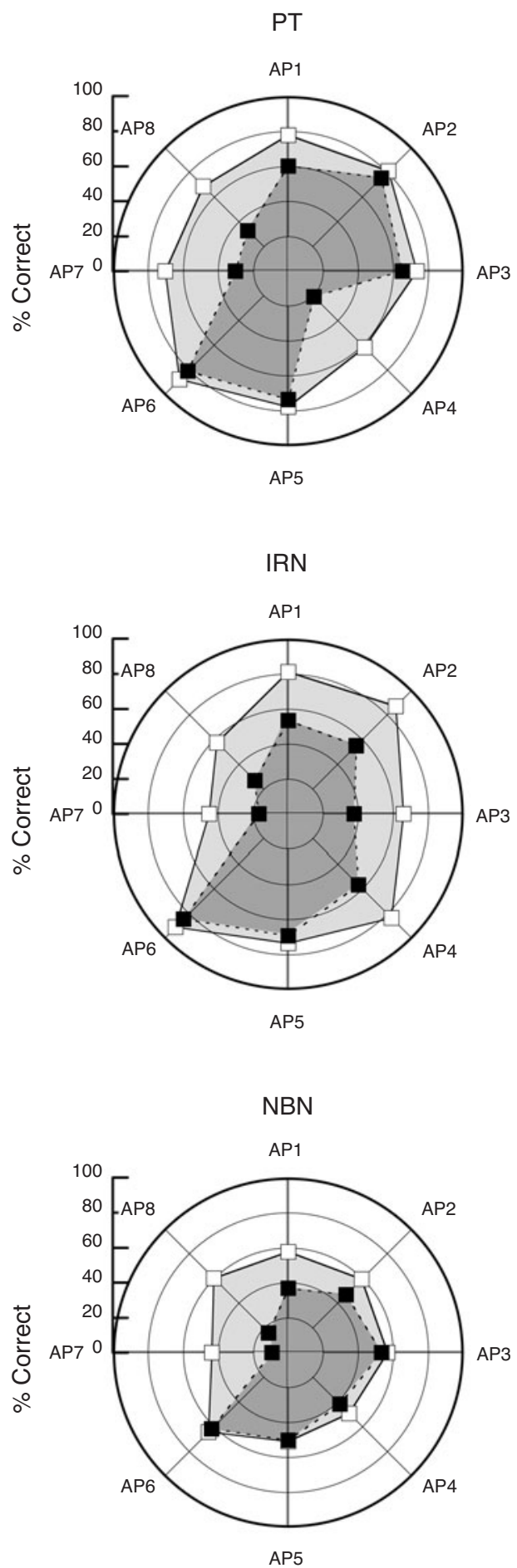
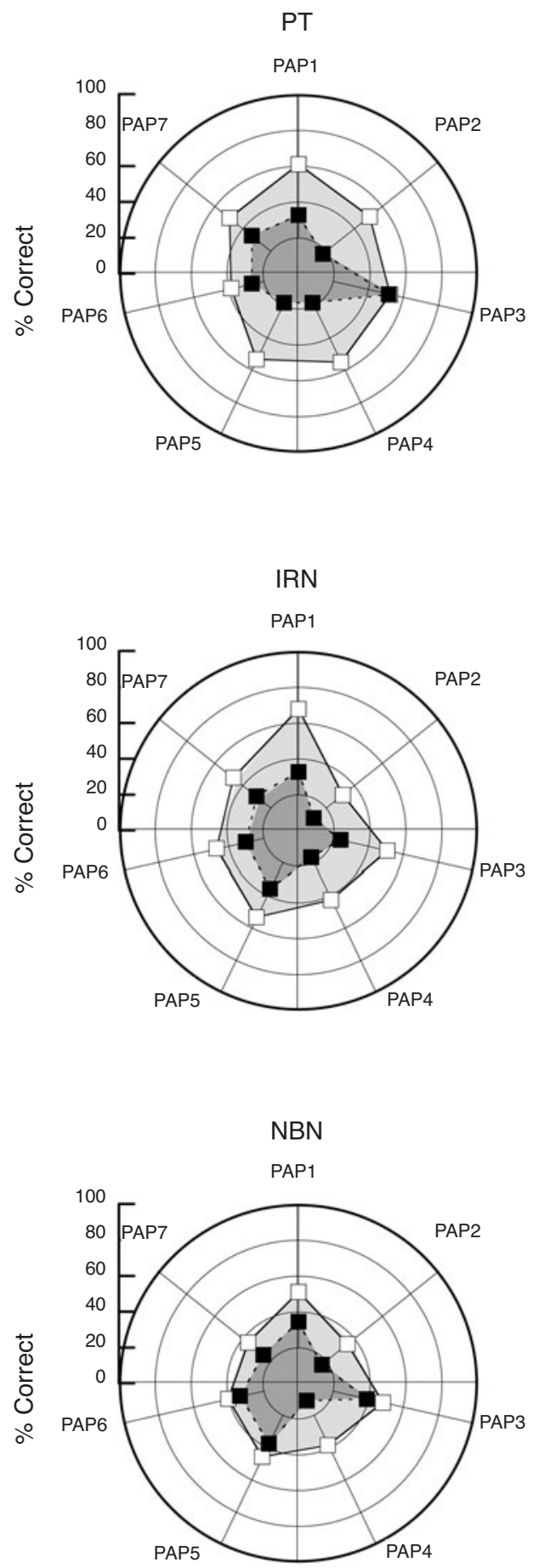

$\square-$ chroma

Figure 8. Individual differences in the absolute pitch (AP, left circles) and partial absolute pitch (PAP, right circles) groups in Experiment 2. The scores from each group are shown as one circle each for pure tone (PT), iterated rippled noise (IRN), and narrowband noise (NBN) stimuli. Each axis represents the percentage of correct responses for each subject. The differences between the chroma scores and the chroma and height scores represent the octave errors. 
gesting different individual weightings of place cues in musical pitch identification.

\section{GENERAL DISCUSSION}

This study indicated that temporal cues are important for chroma identification by AP possessors and that place cues are important for height judgment by both AP and NAP possessors. There was a lot of variability in the data, and the number of subjects in each group was low. These may limit the generality of the findings. However, at least for the data in the present study, the differences were significant.

One possible interpretation of these findings is that musical experience may incline one to use both temporal and place cues in musical pitch identification but that, when the weight on the temporal cues is emphasized, AP ability may be acquired. When a listener perceives chroma, or musical intervals, such as an octave or a perfect fifth, it is quite natural to assume that the temporal cue is particularly important (Ohgushi, 1983). Notes with an interval such as an octave (frequency ratio, $1: 2$ ) or a perfect fifth (frequency ratio, 2:3) are similar from the viewpoint of the periodicity of the auditory nerve firing, but these frequency ratios may not be especially significant from the viewpoint of the position on the basilar membrane. Furthermore, the upper limit of chroma perception of AP possessors is about $5 \mathrm{kHz}$ (Bachem, 1948), which almost agrees with the limit of phase locking (Ruggero, 1992). This may reflect the importance of temporal cues in chroma perception.

A hypothesis can now be made. Children who pay more attention to temporal cues than to place cues and learn the connection between temporal cues and note names may acquire AP. The tendency to use temporal cues for identifying chroma may be enhanced if teachers put a stronger emphasis on chromatic structures than on the absolute height of musical pitch when they teach note names at the early stages of children's music experience. This hypothesis is consistent with the idea that AP, instead of being a rare or somewhat mysterious ability, as is generally believed, is actually possessed by many people to some extent (Levitin, 1994). Evaluating this hypothesis requires further studies from various viewpoints that take into account the development of neural systems and the plasticity of auditory functions.

In this study, we found that AP possessors are able to use temporal cues more effectively in identifying musical chroma, but exactly how they are able to do this remains unclear. Does the difference from NAP possessors consist of the ability to detect temporal cues? Or does the difference lie not in detection ability, but in the process by which temporal cues are used for pitch name identification? Clarifying these points will require further studies in which the discrimination and detection abilities of place and temporal cues are examined.
There was a limitation on the stimuli in this study. To manipulate place and temporal cues separately, we used NBNs and IRNs. NBNs provide strong place cues but less salient temporal cues, and IRNs provide strong temporal cues and less salient place cues. However, we were unable to manipulate place and temporal cues so that they would be perfectly independent from each other. NBNs contain temporal cues, although they are less salient, and IRNs contain spectral ripples that provide place cues. However, when frequencies are high, these ripples cannot be resolved in the auditory filters, so these ripples of IRNs may have little effect on the results. Manipulating place and temporal cues so that they are perfectly independent from each other may not be possible. The solution would be to find stimuli that separate place and temporal cues more strongly. ${ }^{1}$

\section{REFERENCES}

BACHem, A. (1937). Various types of absolute pitch. Journal of the Acoustical Society of America, 9, 146-151.

BACHEM, A. (1948). Chroma fixation at the ends of the musical frequency scale. Journal of the Acoustical Society of America, 20, 704-705.

BACHEM, A. (1955). Absolute pitch. Journal of the Acoustical Society of America, 27, 1180-1185.

Benguerel, A. P., \& Westdal, C. (1991). Absolute pitch and the perception of sequential musical intervals. Music Perception, 9, 105120.

BRADY, P. T. (1970). Fixed-scale mechanism of absolute pitch. Journal of the Acoustical Society of America, 48, 883-887.

CUDDY, L. L. (1968). Practice effects in the absolute judgment of pitch. Journal of the Acoustical Society of America, 43, 1069-1076.

FUJISAKI, W., \& KASHINO, M. (2002). The basic hearing abilities of absolute pitch possessors. Acoustical Science \& Technology, 23, 77-83.

Klein, M., Coles, M. G. H., \& Donchin, E. (1984). People with absolute pitch process tones without producing a P300. Science, 223, 1306-1309.

LEVITIN, D. J. (1994). Absolute memory for musical pitch: Evidence from the production of learned melodies. Perception \& Psychophysics, 56, 414-423.

MIYAZAKI, K. (1993). Absolute pitch as an inability: Identification of musical intervals in a tonal context. Music Perception, 11, 55-72.

MiYAZAKI, K. (1995). Perception of relative pitch with different references: Some absolute pitch listeners can't tell musical interval names. Perception \& Psychophysics, 57, 962-970.

MIYAZAKI, K., \& RAKOWSKI, A. (2002). Recognition of notated melodies by possessors and nonpossessors of absolute pitch. Perception \& Psychophysics, 64, 1337-1345.

MOORE, B. C. J. (1997). An introduction to the psychology of hearing (4th ed.). London: Academic Press.

OHgushi, K. (1983). The origin of tonality and a possible explanation of the octave enlargement phenomenon. Journal of the Acoustical Society of America, 73, 1694-1700.

ProfitA, J., \& BIDDER, T. G. (1988). Perfect pitch. American Journal of Medical Genetics, 29, 763-771.

RugGero, M. A. (1992). Physiology and coding of sound in the auditory nerve. In A. N. Popper \& R. R. Fay (Eds.), The mammalian auditory pathway: Neurophysiology (pp. 34-93). New York: SpringerVerlag.

SIEGEL, J. A. (1972). The nature of absolute pitch. In I. E. Gordon (Ed.), Studies in the psychology of music (Vol. 8, pp. 65-89). Iowa City: University of Iowa Press.

Takeuchi, A. H., \& Hulse, S. H. (1993). Absolute pitch. Psychological Bulletin, 113, 345-361. 
Wayman, J. W., Frisina, R. D., Walton, J. P., Hantz, E. C., \& CrumMER, G. C. (1992). Effects of musical training and absolute pitch ability on event-related activity in response to sine tones. Journal of the Acoustical Society of America, 91, 3527-3531.

Yost, W. A. (1996). Pitch of iterated rippled noise. Journal of the Acoustical Society of America, 100, 511-518.

ZATORRE, R. J. (2003). Absolute pitch: A model for understanding the influence of genes and development on neural and cognitive function. Nature Neuroscience, 6, 692-695.

\section{NOTE}

1. The IRNs used in this study have frequency regions higher than the NBNs. This results in an additional timbre difference between IRNs and NBNs, which might be part of the reason for the differences obtained for IRN stimuli.

(Manuscript received January 14, 2003; revision accepted for publication June 2, 2004.) 